\title{
Research Paper: \\ Effect of Toe Only Rocker at 10 and 15 Degrees on Balance and Walking Speed in Elderly Adults
}

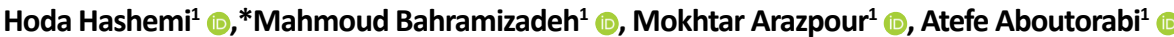

1. Department of Orthotics and Prosthetics, School of Rehabilitation Sciences, University of Social Welfare and Rehabilitation Sciences, Tehran, Iran.

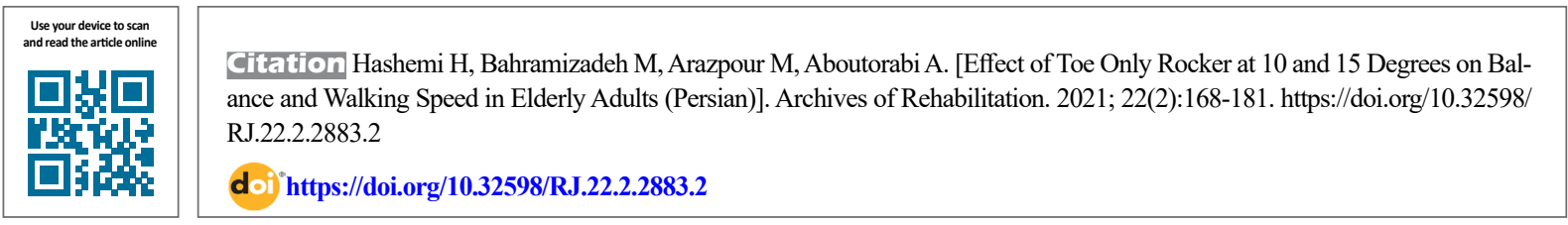

Received: 22 Aug 2020 Accepted: 01 Oct 2020 Available Online: $01 \mathrm{Jul} 2021$

Keywords: Balance, Walking speed, Elderly, Rocker sole, Toe-only rocker

\begin{abstract}
Objective Balance is one of the indicators to determine independence in performing daily activities in the elderly. One of the influential factors in postural control and balance is walking speed. This study aims to evaluate the effect of rocker soles at two degrees of 10 and 15 on the walking speed and balance of the elderly.

Materials \& Methods The study participants were 19 older adults aged 60 years or older (13 women and 6 men; mean age $=66.1$ years, mean height $=1.63 \mathrm{~m}$, mean weight $=70.3 \mathrm{~kg})$. Three models of shoes were used: shoes with a 10-degree rocker sole angle, shoes with a 15-degree rocker sole angle, and control shoes. Walknig speed was evaluated with $10-\mathrm{m}$ walking test, while balance and dynamic postural control were assessed with the Berg balance Scale (BBS) and Star Excursion balance test, respectively. The Shapiro-Wilks test was used to examine the normality of data distribution, and repeated-measures ANOVA and Wilcoxon test were used to compare the effects of different rocker angles on balance and walking speed. The obtained data were analyzed in SPSS v. 22.

Results There was no significant difference in walking speed $(P=0.993)$, dynamic postural balance at anterior $(P=0.835)$, posterolateral $(P=0.86)$, and posteromedial $(P=0.598)$ directions and balance obtained from $B B S(P=0.625)$ among the groups using three shoe models.

Conclusion It seems that the use of rocker sole shoes does not affect the balance and walking speed of the elderly. This study supports the administration of shoes with toe-only rocker soles to the elderly.
\end{abstract}

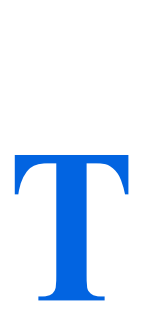

Extended Abstract

\section{Introduction}

he world's elderly population has grown significantly over the past few decades [1-3] and is expected to increase in the future [2]. With aging, all factors involved in balance, including walking speed [1], are affected [4]. About $27 \%-65 \%$ of the elderly experience falls at least once a year $[5,6]$. Fear of falling is the most common fear among the elderly $[7,8]$. Several interventions have been used to maintain and improve walking speed and balance in the elderly. These interventions include vestibular neurosurgery, physiotherapy, exercise therapy, orthosis interventions, and using special shoes [11-15]. Since the feet have direct contact with the ground during walking, any change in the space between the sole and the ground can affect the postural stability of people $[9,10]$. Many features in shoe design can affect balance and walking speed [11-13]. In assessing the characteristics of the sole of the shoes, one of the common interventions used for a wide range of problems

\section{* Corresponding Author:}

Mahmoud Bahramizadeh, PhD.

Address: Department of Orthotics and Prosthetics, School of Rehabilitation Sciences, University of Social Welfare and Rehabilitation Sciences, Tehran, Iran Tel: +98 (912) 2483320

E-Mail: mbzoandp@gmail.com 
and target groups is the addition of rocker soles to the shoes $[14,15]$. Other rockers have various therapeutic effects on the target groups [19]. The geometric characteristics of toe rockers in the anterior part are determined by three variables of apex angle, apex position, and rocker angle [21]. The rise and function of the apex change the rate of movement of the lower extremity joints, especially the ankle, walking speed, kinematic gait, and the patterns of rocker during the gait cycle. In this way, a change in the apex angle and position can improve or weaken these variables $[21,22]$ and, thus, affect people's balance and walking speed.

In examining the characteristics of the toe rocker, Chapman's study showed that the use of a rocker with an apex angle of 95-90 degrees had good equilibrium effects on the study groups [16]. Meyer et al. and van Schie et al.' studies showed that, for optimal balance performance, the most effective apex position is at $55 \%-65 \%$ of the shoe length [17, 18]. In another study, the effect of rocker angles on the degree of dorsiflexion and toe clearance was investigated. The results showed that, regardless of the ground inclination, shoes with toe rockers at angles of 10-15 degrees compared to other rockers significantly increased toe clearance in the elderly and consequently reduced the risk of falling [19].

Given the growing rate of the aging population and the increasing injuries caused by poor postural balance, a study in the field of balance and walking speed of the elderly can be of great value to this group. The use of rocker sole shoe is one of the common interventions for increasing muscle strength in the elderly and young people. Despite the few studies on the effect of rockers in the elderly population, some have concluded that adding a rocker to the sole of the shoe can improve muscle strength in the long term and, thereby, improve postural stability. Achieving the desired effects on muscle strength requires rocker use on the sole for at least 6 months [20-22]. However, there is a concern that during this 6-month period, older people may have balance problems and slow walking. Thus, the present study aims to evaluate the short-term effect of toe rockers with specific angles on walking speed and postural control of the elderly.

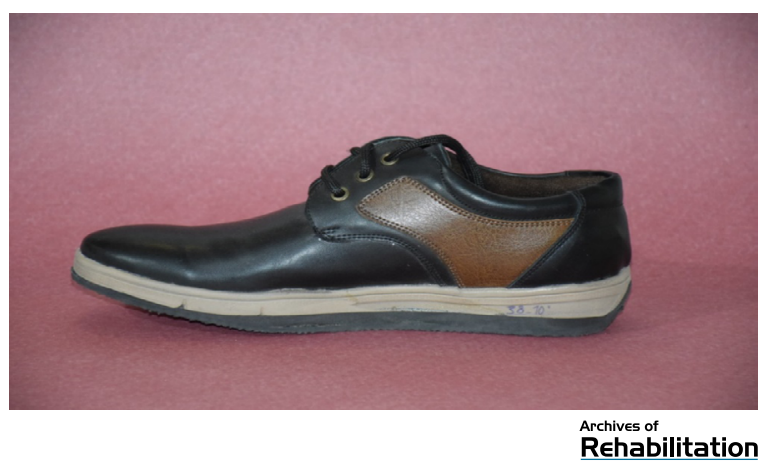

Figure 1. Test shoe model with a rocker angle of 10 degrees

\section{Materials and Methods}

This research is a quasi-experimental study with a pretestposttest design. The inclusion criteria were being healthy and older than 60 years, while the exclusion criteria were having neuromuscular diseases, peripheral neuropathy, diabetes, acute musculoskeletal injuries, acute pain in the lower limbs and lower back, using walking aids, balance problems, and acute heart or lung diseases. To determine the sample size, first, a pilot study was performed on five older people. Assuming an effect size of 0.5 , the minimum sample size was 19 to achieve a test power of 0.86 . The participants' dynamic postural stability was assessed by the Star Excursion Balance Test (SEBT). It is a reliable test with an intraclass correlation coefficient of 0.89-0.93 and a coefficient of variation of 0.3-4.6 [23]. To normalize the data, the SEBT results were divided by the leg length, and their mean values were used in the analysis [24]. The balance of participants was evaluated using the Persian version of the Berg Balance Scale (BBS), whose psychometric properties have been evaluated in a previous study [25]. The total score of BBS is 56, which shows the highest balance ability. The internal and external reliabilities of this test in the elderly are 0.98 and 0.99 , respectively [26]. The walking speed was assessed using the 10 -m walking test where the participant walks a distance of $20 \mathrm{~m}$ at a safe speed. The test was repeated 3 times, and the best record was set as the test score [27].

Ethyl vinyl acetate rubber was used to prepare the toe rocker in shoes with similar insoles and soles. The location of the rocker head was at $65 \%$ of the shoe length [28]. To maintain a safe environment for the elderly, the rocker angles were set at the maximum values, which did not increase the risk of falls in the elderly in previous studies [19, 29]. In the first test model, the rocker angle was 10 degrees (Figure 1), and in the second test model, it was 15 degrees (Figure 2). The shoe's sole was completely thick at the heel, at $65 \%$ of the shoe length. A shoe model similar to the test shoes, but different only in type and thickness of the sole, was used as the control shoe (Figure 3). To analyze the data,

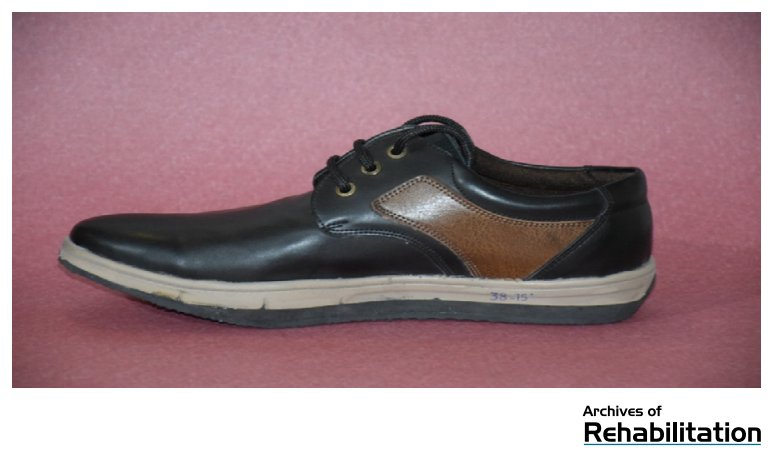

Figure 2. Test shoe model with a rocker angle of 15 degrees 


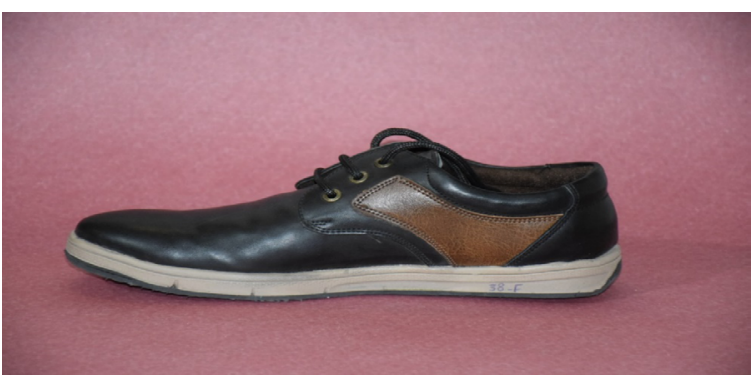

Figure 3. Control shoe model

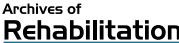

first, the normality of their distribution was examined using the Shapiro-Wilk test, whose results showed a normal distribution for all data $(\mathrm{P}=0.2)$ except for the BBS data $(\mathrm{P}<0.001)$. Therefore, ANOVA and the Wilcoxon test were used to analyze the collected data.

\section{Results}

Out of 19 participants, 6(31.6\%) were men, and 13(68.4\%) were women. Their age ranges, mean of height, weight, Body Mass Index (BMI), and lower limb length ranges are presented in tables 1, 2, and 3. The Mean \pm SD BBS score in the control shoe group was $55.46 \pm 0.96$, ranging from 52 to 56 . This score was $55.21 \pm 0.98$ in the shoe group with a 10-degree toe rocker and $55.26 \pm 1.05$ in the shoe group with a 15-degree toe rocker; both ranged from 52 to 56 . The result of the Wilcoxon test showed no statistically significant difference between the three groups regarding this variable $(\mathrm{P}=0.625)$. The Mean \pm SD score of the 10 - $\mathrm{m}$ walking test in the control group was $1.14 \pm 0.24 \mathrm{~m} / \mathrm{s}$, ranging from 0.59 to $1.6 \mathrm{~m} / \mathrm{s}$. This score was $1.15 \pm 0.18 \mathrm{~m} / \mathrm{s}$ in the shoe group with 10-degree toe rocker (range: 0.83 to $1.38 \mathrm{~m} / \mathrm{s}$ ) and $1.15(0.22) \mathrm{m} / \mathrm{s}$ in the shoe group with 15 -degree toe rocker (range: 0.73 to $1.72 \mathrm{~m} / \mathrm{s}$ ). The result of the Wilcoxon test showed no statistically significant difference between the three groups regarding this variable $(\mathrm{P}=0.993)$. The result of the Wilcoxon test showed no statistically significant difference in SEBT score between the three groups (Table 4).

\section{Discussion and Conclusion}

The present study revealed that adding a toe rocker with angles of 10 or 15 degrees had no adverse effect on the balance and the walking speed of the elderly. The use of two rocker angles with certain values was based on previous studies, which showed that these rocker angles positively affected toe clearance during the swing phase in the elderly $[19,29]$. Wearing rocker shoes reduces the person's awareness of foot conditions by affecting the movement of the ankle joints and reducing the level of reliance [30]. Thus, due to compensatory mechanisms and caution, the travel distance in SEBT might be reduced after using rocker shoes compared to control shoes. However, the participants traveled relatively the same distance with all three shoe models. One of the reasons for the lack of significant differences in the results can be an increase in the person's muscle activity to achieve a stable condition. Ghomian et al. [25], in a study on 17 patients with diabetic neuropathy, stated that when wearing rocker shoes, muscular strength increases in response to anterior and posterior stimuli to reach a stable

Table 1. Average age distribution of the participants

\begin{tabular}{ccccc}
\hline Age Range (y) & N & \% & Cumulative \% \\
\hline $60-69$ & 15 & 78.9 & 78.9 \\
$70-79$ & 3 & 15.8 & 94.7 \\
$\geq 80$ & 1 & 5.3 & 100 \\
\hline Total & 19 & 100 & - \\
\hline
\end{tabular}

Table 2. Mean \pm SD values of height, weight, and Body Mass Index (BMI) in the participants

\begin{tabular}{rccc}
\hline Variables & Mean \pm SD & Min. & Max. \\
\hline Height $(\mathrm{m})$ & $1.63 \pm 0.09$ & 1.5 & 1.85 \\
Weight $(\mathrm{kg})$ & $70.3 \pm 11.5$ & 45 & 85 \\
BMl $\left(\mathrm{kg} / \mathrm{m}^{2}\right)$ & $26.41 \pm 3.08$ & 18.73 & 31.25 \\
\hline
\end{tabular}


Table 3. The average length of the lower limbs of the participant

\begin{tabular}{cccc}
\hline Lower Limb Length $(\mathbf{c m})$ & $\mathbf{N}$ & $\%$ & Cumulative \% \\
\hline $70-75$ & 10 & 52.7 & 52.7 \\
$76-80$ & 5 & 26.3 & 78.5 \\
$81-85$ & 2 & 10.5 & 89.5 \\
$86-90$ & 2 & 10.5 & 100 \\
\hline Total & 19 & 100 & - \\
\hline
\end{tabular}

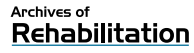

condition. In other words, the lack of change in postural stability while wearing rocker shoes can be due to increased muscle activity and the person's effort to maintain a stable posture. In their study, no significant difference was observed between shoes with toe rockers and control shoes regarding postural stability [31]. Brenton-Rule et al. evaluated the walking footwear on the postural stability of 21 healthy older adults. They also found no significant difference between the use of rocker shoes and regular walking shoes [32]. Ramstrand's study of 31 women over the age of 50 showed that eight weeks of wearing MBT (Masai Barefoot Technology) shoes had no significant effect on the static stability of participants [21]. At the same time, the results of Albright et al. [44], Demura et al. [45], and Arazpour et al. [46] are contrary to our results. Albright et al. used shoes with rocker bottom soles, Demura et al. used shoes with rounded soft soles, and Arazpour et al. used shoes with heel-to-toe rocker soles. The discrepancy in results may also be due to the difference in the postural stability system between the elderly and young people. In all three studies, the participants were 20-25 years old, while the participants in our study were 66 years or older.

The results obtained from the $10-\mathrm{m}$ walking test showed that the use of shoes with a 10- or 15-degree toe rocker did not cause a significant change in the walking speed of the elderly. Adding a rocker to the sole of the shoe increases the activity of ankle plantar flexors. If the rocker material is hard, it will prevent metatarsal joint movement, and, hence, the so-called metatarsal bone fracture will not occur. This status will increase the moment arm of plantar flexor muscles and eventually needs extra effort to lift the heel off the ground $[36,37]$. On the other hand, the addition of a toe rocker increases the angle of hip extension in the middle and end of the static phase; as a result, the step length decreases [38]. At a certain distance, if the step length decreases and the cadence increases, the walking speed will not change. Forgani et al. and Arazpour et al. reported the same walking speed of the participants using control shoes and rocker

Table 4. Mean \pm SD scores of the Star Excursion Balance Test (SEBT) at three directions

\begin{tabular}{|c|c|c|c|c|c|}
\hline \multicolumn{3}{|c|}{ Control Shoe Group } & $\begin{array}{c}\text { Shoe Group With a 10-Degree } \\
\text { Toe Rocker }\end{array}$ & $\begin{array}{c}\text { Shoe Group With a 15-Degree } \\
\text { Toe Rocker }\end{array}$ & $\begin{array}{c}\text { Test } \\
\text { Results }\end{array}$ \\
\hline \multirow{3}{*}{$\begin{array}{l}\text { SEBT at the anterior } \\
\text { direction }\end{array}$} & Mean $\pm S D$ & $0.78 \pm 0.12$ & $0.79 \pm 0.09$ & $0.8 \pm 0.07$ & \multirow{3}{*}{$P=0.826$} \\
\hline & Min. & 0.6 & 0.66 & 0.69 & \\
\hline & Max. & 1.04 & 0.98 & 1 & \\
\hline \multirow{3}{*}{$\begin{array}{l}\text { SEBT at the postero- } \\
\text { medial direction }\end{array}$} & Mean $\pm S D$ & $0.82 \pm 0.08$ & $0.83 \pm 0.11$ & $0.82 \pm 0.1$ & \multirow{3}{*}{$P=0.835$} \\
\hline & Min & 0.69 & 0.62 & 0.71 & \\
\hline & Max & 0.97 & 1.09 & 1.04 & \\
\hline \multirow{3}{*}{$\begin{array}{l}\text { SEBT at the postero- } \\
\text { lateral direction }\end{array}$} & Mean $\pm S D$ & $0.66 \pm 0.14$ & $0.65 \pm 0.1$ & $0.69 \pm 0.11$ & \multirow{3}{*}{$P=0.598$} \\
\hline & Min. & 0.41 & 0.44 & 0.49 & \\
\hline & Max. & 0.97 & 0.92 & 0.88 & \\
\hline
\end{tabular}


shoes $[35,39]$. Similar results were also observed in studies by Meyer et al. and Van Bogart $[15,38]$. However, they suggested that the reason for the same speed when walking with rocker shoes was the increase of cadence and the decrease of stride length. One of the confounding variables and limitations of this study was the difference in the sole thickness in rocker-soled and control shoes. Although the effect of increasing the sole thickness on the weight of the shoes is statistically insignificant, the difference in the effect of the sole thickness on the balance is still debated. Since the results of clinical trials depend on the carefulness of the examiner and the location of the tests, all tests were performed in a place with standard conditions provided by an orthotist. It is recommended that other temporal and spatial parameters of walking in the elderly be evaluated in future studies by using rocker shoes.

\section{Ethical Considerations}

Compliance with ethical guidelines

All ethical principles are considered in this article. The participants were informed about the purpose of the research and its implementation stages. They were also assured about the confidentiality of their information and were free to leave the study whenever they wished, and if desired, the research results would be available to them.

Funding

This research did not receive any grant from funding agencies in the public, commercial, or non-profit sectors.

\section{Authors' contributions}

Conceptualization, methodology, software, validation, formal analysis, investigation, resources, data curation, writing - original draft preparation, writing - review \& editing, visualization: All authors; Supervision: Mahmoud Bahramizadeh, Mokhtar Arazpour, Atefe Aboutorabi.

\section{Conflict of interest}

The authors declared no conflict of interest. 


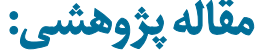 \\ ثأثير راكر ينجه با زواياى ^ا و ه 1 درجه بر تعادل و سرعت راه رفتن القراد سالمند

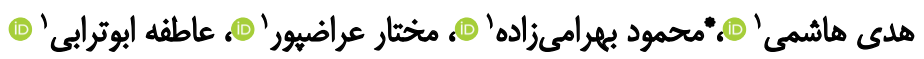

ا. كروه ارتوز و يروتز، دانشكده علوم توانبخشى، دانشكاه علوم توانبخشى و سلامت اجتماعى، تهران، ايران.

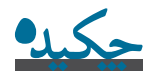

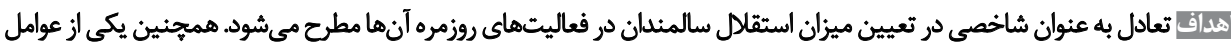

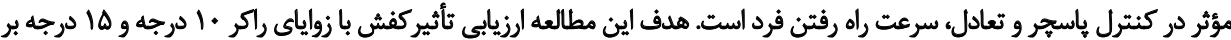
تعادل و سرعت راه رفتن سالمندان است ونتر

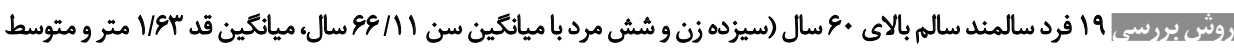

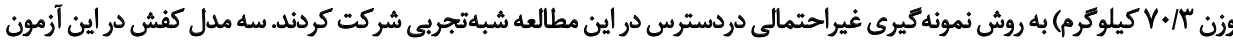

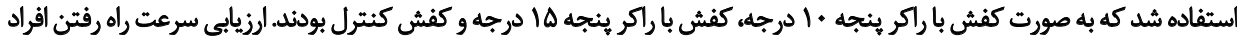

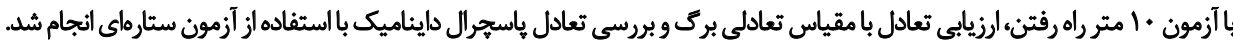

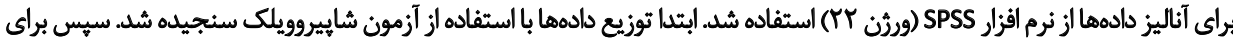

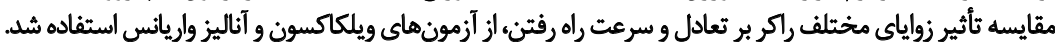

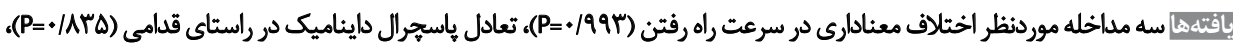

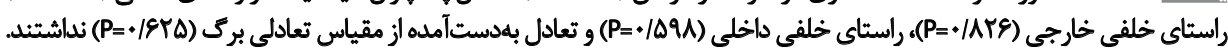

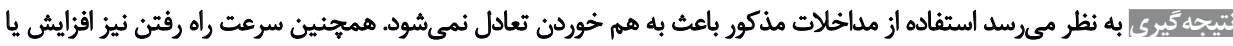

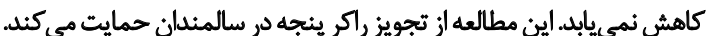

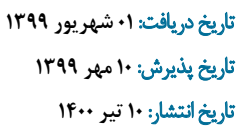

كليدوأوهها:

تعادل، سرعت راه رفتن، سالمند، كفش راكر دارة،

راكر ينجه كفئ

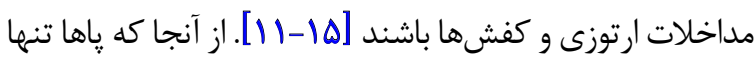

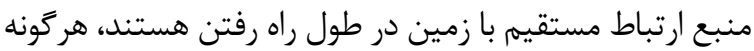

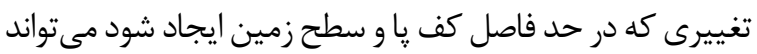

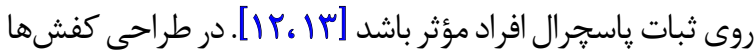

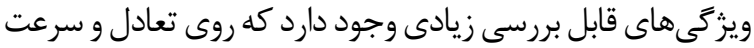

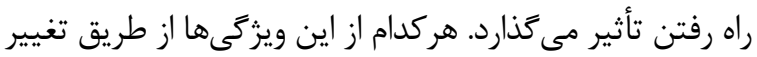

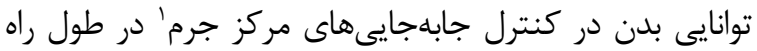

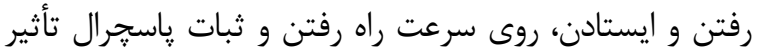

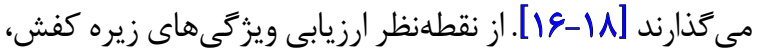

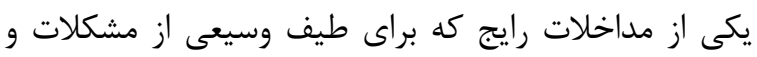

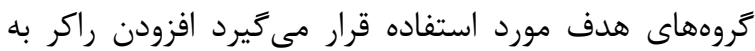

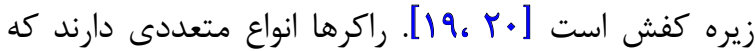

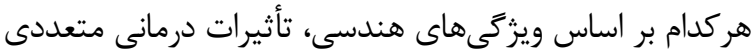

مقدمه

در طول קند دها كذشته جمعيت افراد سالمند در دنيا به إنه

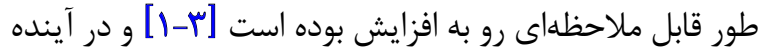

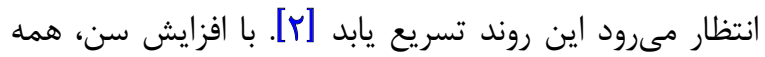

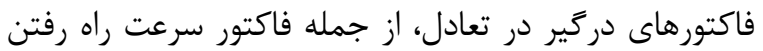

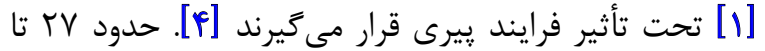

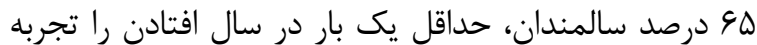

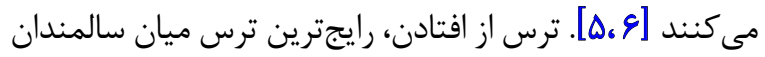

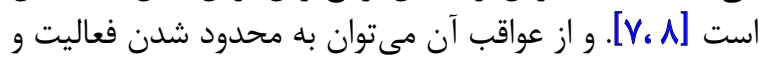

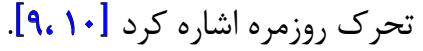
امروزه مداخلات متعددى براى حفظ و ارتقاى سرعت راه رفتر رفتن

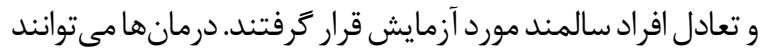
شامل جراحى اعصاب وستيبولار، فيزيوترايى، تمريندرمانى، 
راه رفتن قرار بخيرند. درواقع سوال اصلى اين است كه در دوره

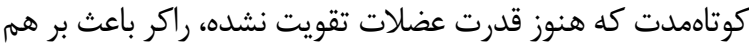

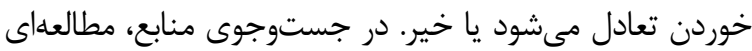

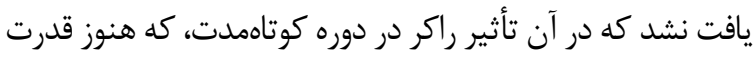

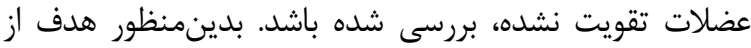

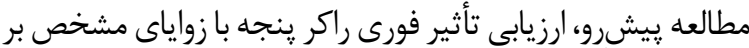

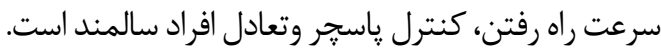

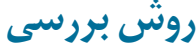

مطالعه از نوع شبالجربى بوده است. انتخاب نمونهها به صورت

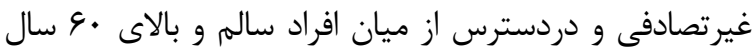

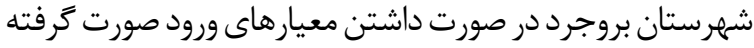

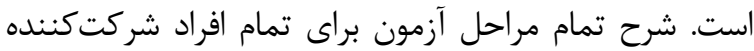

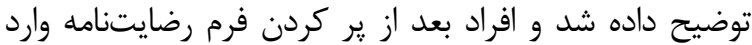

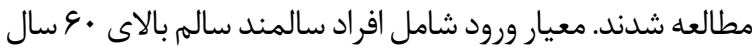

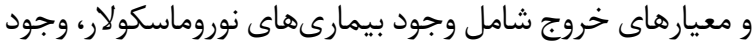

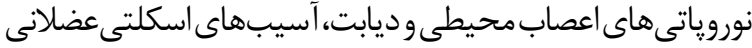

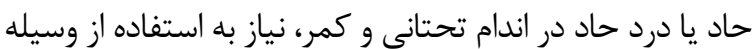

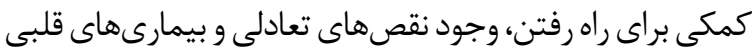

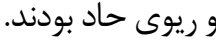

به منظور تعيين حجم نمونه، ابتدا مطالعه پايلوت با نمونا

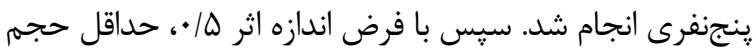

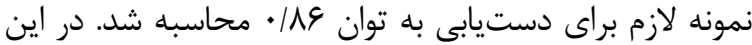
مطالعه حداقل حجم نمونه 9 انفر محاسبه شد ند.

جهت جمع آورى اطلاعات، وزن شركت كنيندكان با استفاده

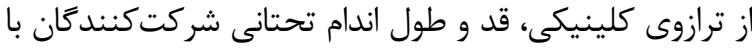

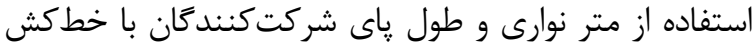

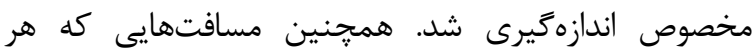

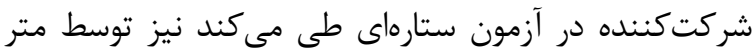

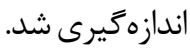

ثبات باسجر ال دايناميك با استفاده از آزمون ستارهاى بررسى

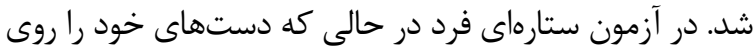

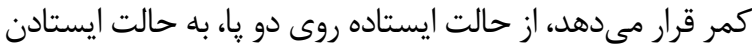

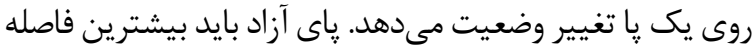

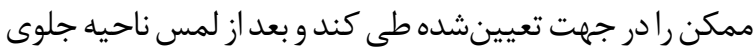

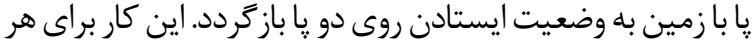

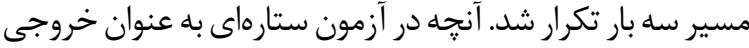

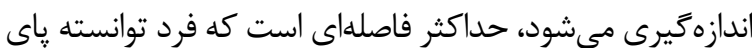

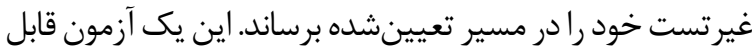

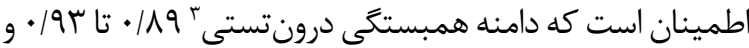

\section{Test-retest intraclass correlations}

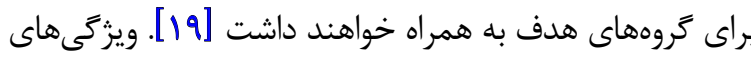

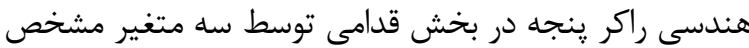

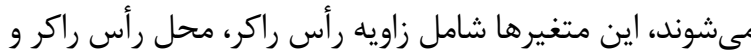

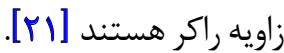

زاويه و محل قرار كيرى رأس راكر در قدام، ميزان حركت مفاصل

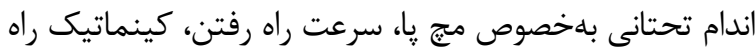

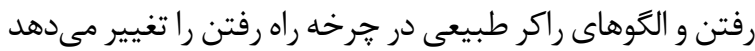

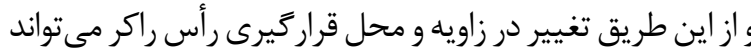

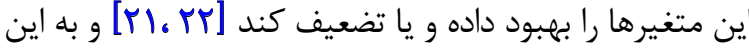
ترتيب تعادل و سرعت راه رفتن افراد راد ادستخوش تغيير كند.

در بررسى ويزگ نىهاى راكر ينجه، مطالعه جِيمن نشان داد كه

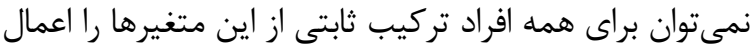

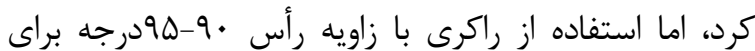

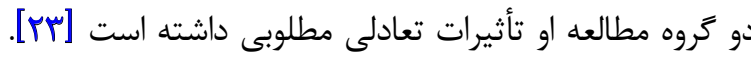

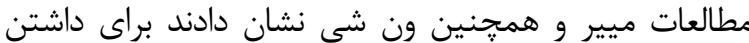

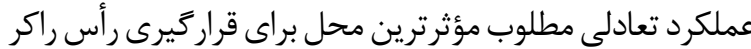

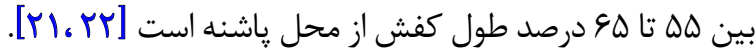
در مطالعهاى، تأثير زواياى راكر روى ميزان دورسىفلكشن و

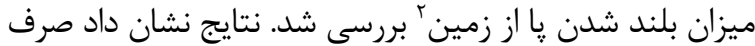

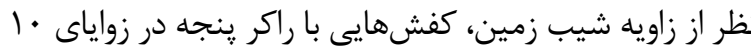

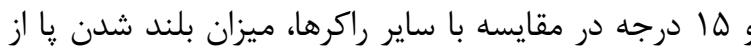

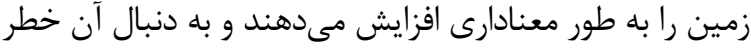

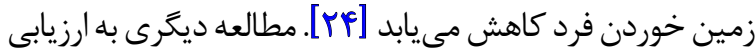

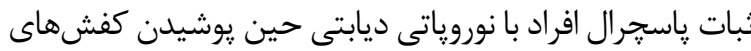

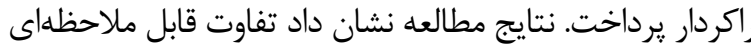

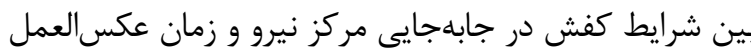

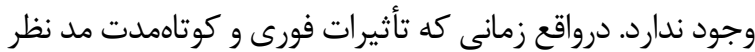

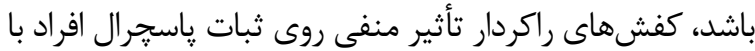

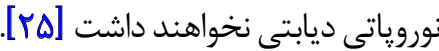
با توجه به نرخ رو به رشد جمعيت سالمندان و افزايش آسيبهاى

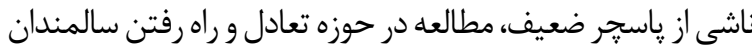

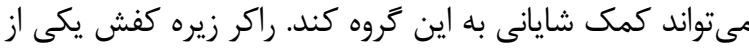

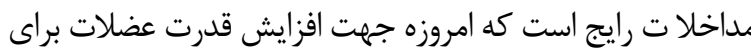

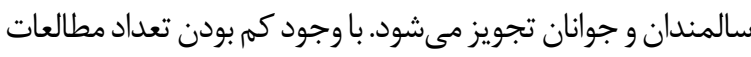

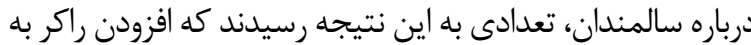

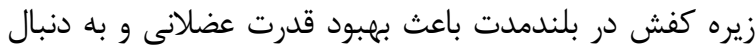

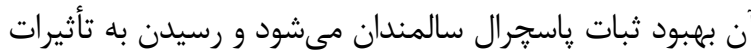

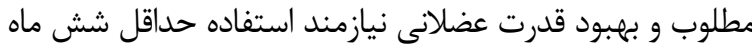

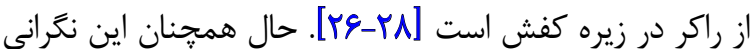

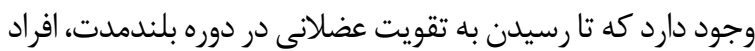

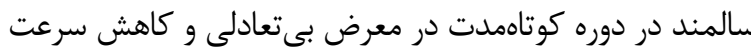

\section{Toe clearance}




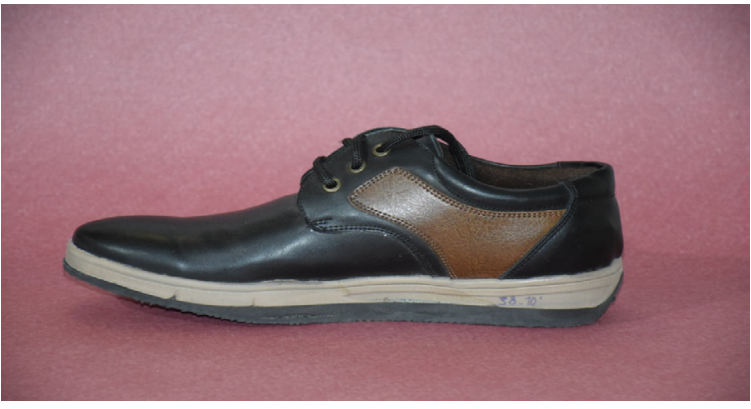

توانبخننى

تصوير ז. كفش با زاويه راكر • لا درجه

اندك بِيشين، خطر زمين خوردن را در سالمندان تقويت نكرده

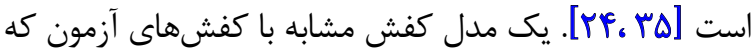

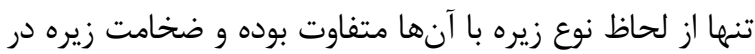

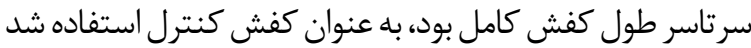

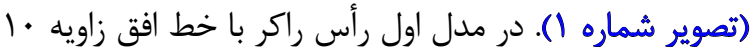

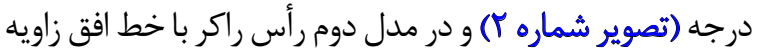

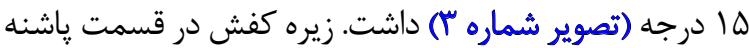

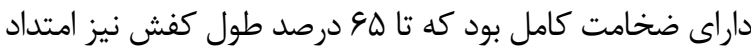

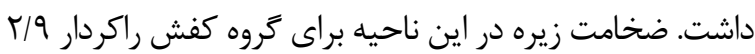

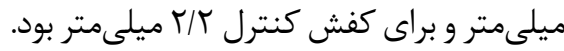

جهت انجام آزمونها، ابتدا آموزشهاى لازم بها افرادشركت كننده

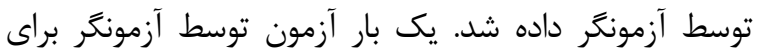

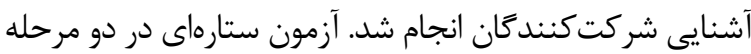

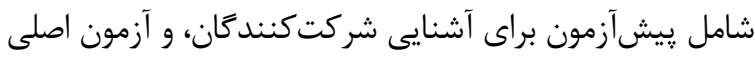

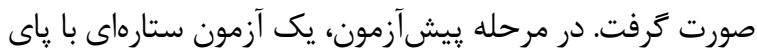

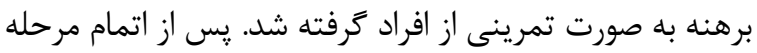
ييش آزمون، ه دقيقه استر احت براى آزمودنى ها در نظر كر كرفته شد.

در مرحله آزمون اصلى، از افراد خواسته شد به منظور آشنا

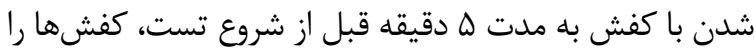

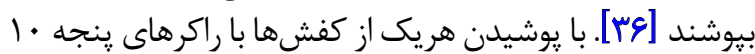

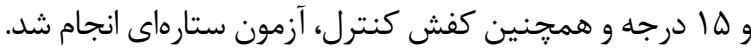

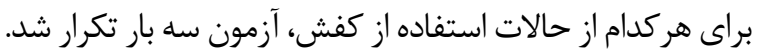

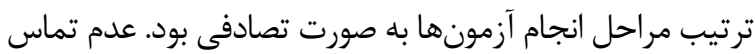

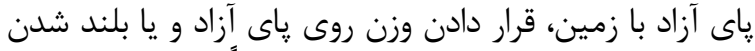

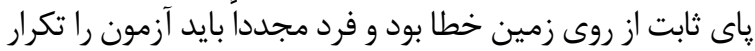

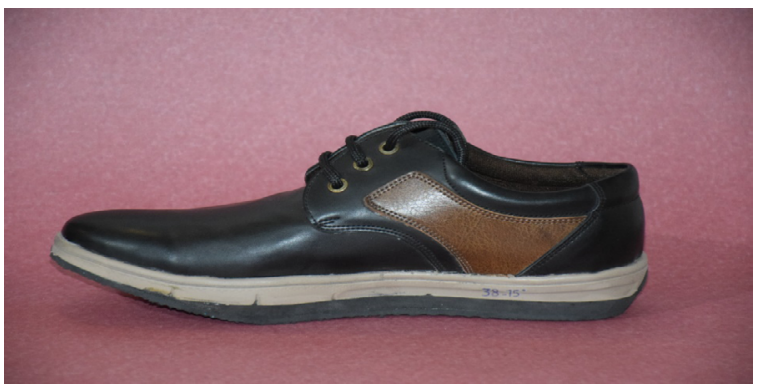

توانبخننى

تصوير "r. كفش با زاويه راكر ها درجه

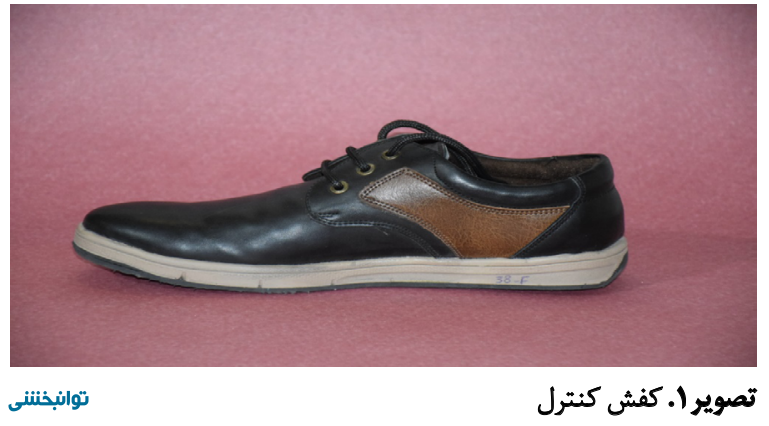

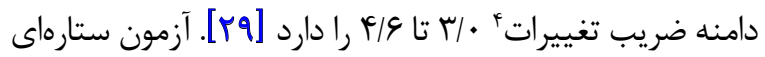

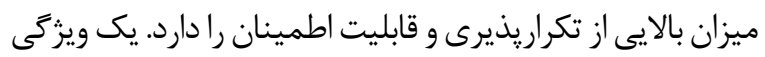

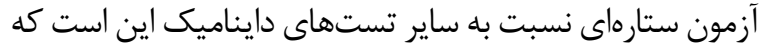

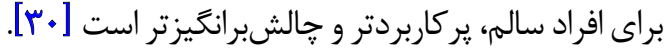
تعادل افر اد با نسخه فارسى مقياس تعادلى برى كه در در تحقيقات

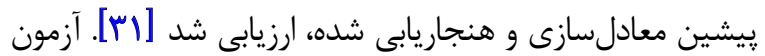

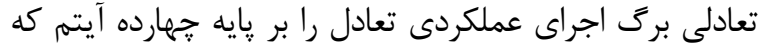

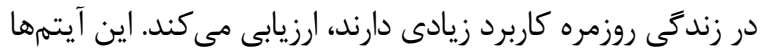

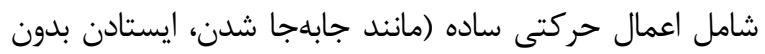

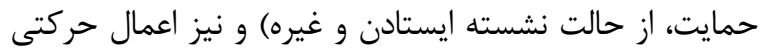

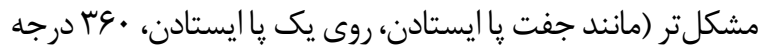

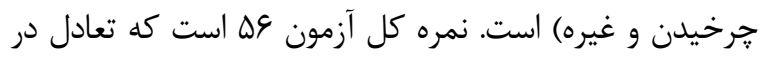

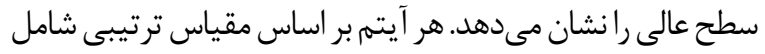

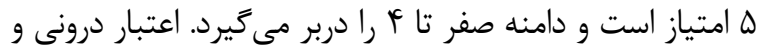

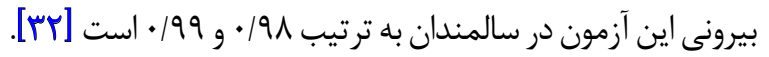

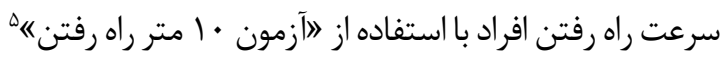

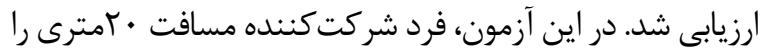

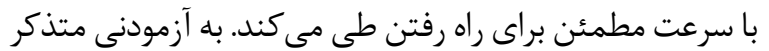

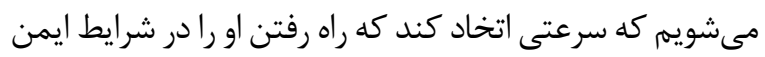

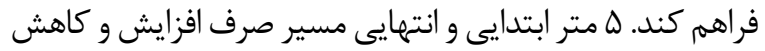

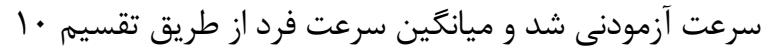

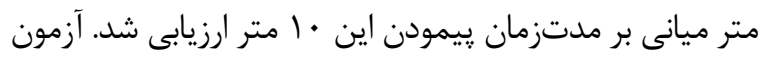

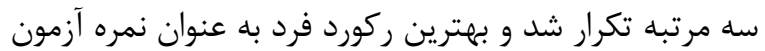

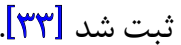

براى انجام اين تحقيق كفشهاى استاندارد با محفظه

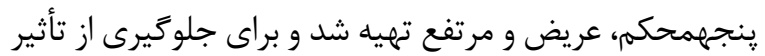

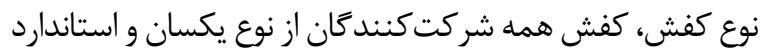

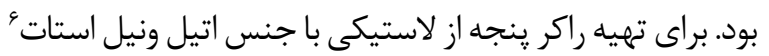

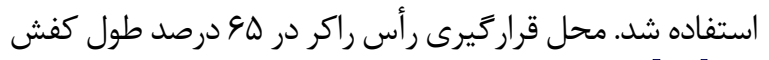

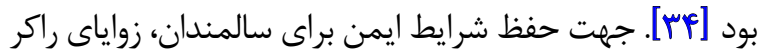
استفادهده در مطالعه ما، حداكثر زوايايى است كه در در مطالعات 


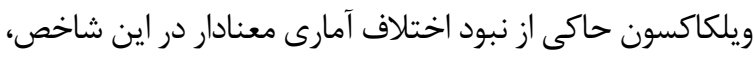

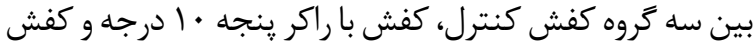

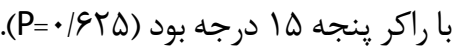

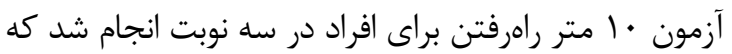

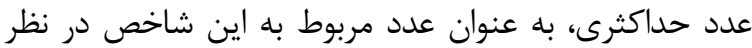

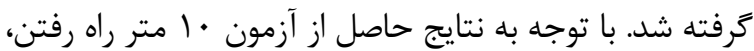

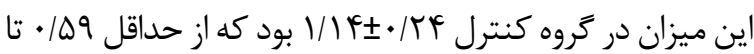

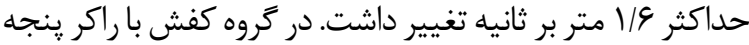

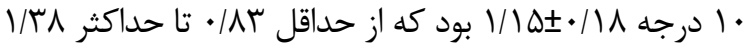

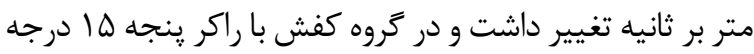

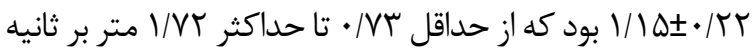

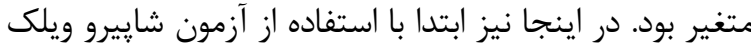

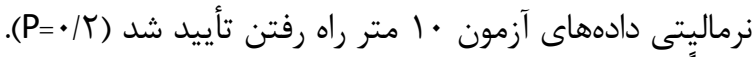

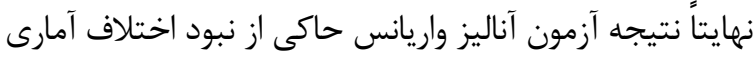

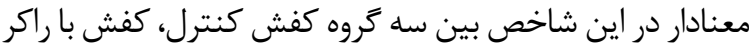

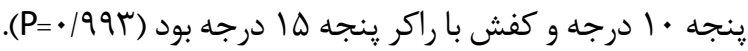

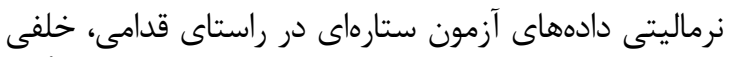

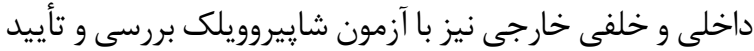

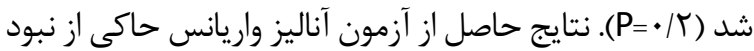

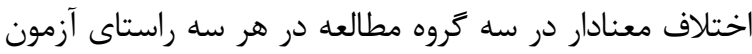

ستارهاى بود (جدول شماره \&).

ب)

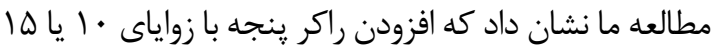

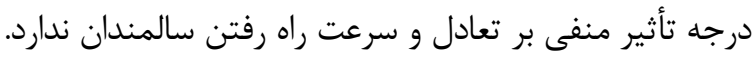

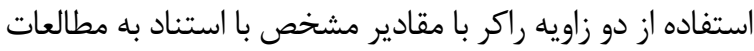

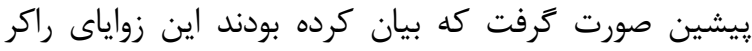

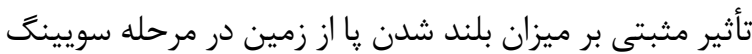

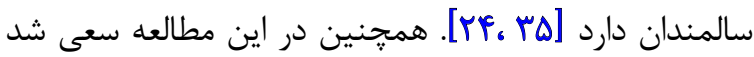

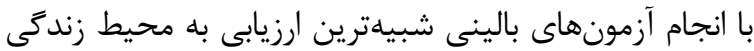

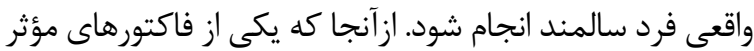

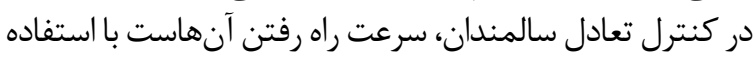
از آزمون • امتر راه رفتن، اين متغير نيز ارزيابى شداه

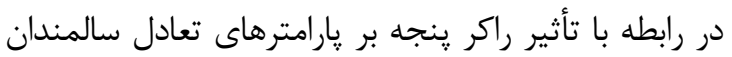

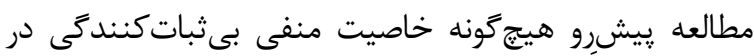

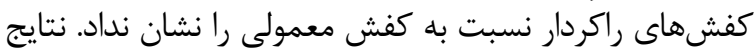

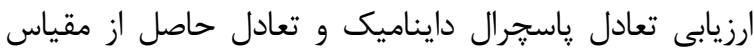

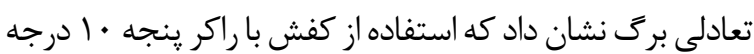

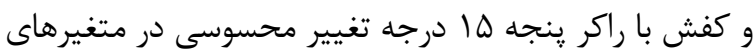

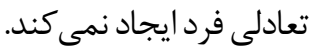

هدف آزمون ستارهاى اين است كه فرد تا حد ممكن تعادل

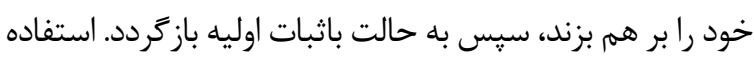

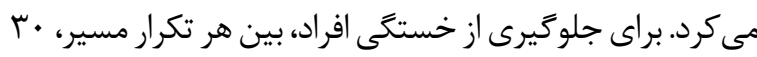

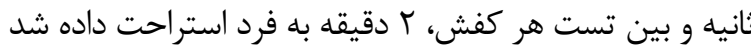

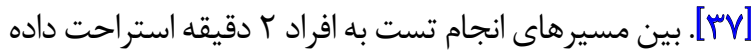

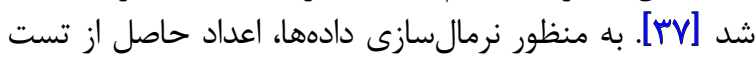

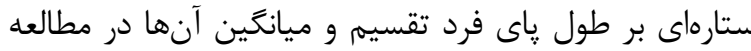

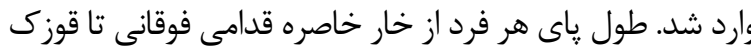

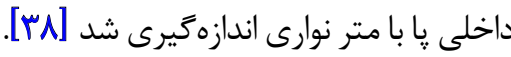

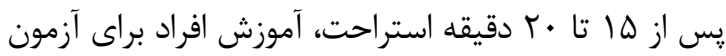

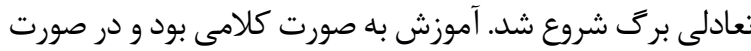

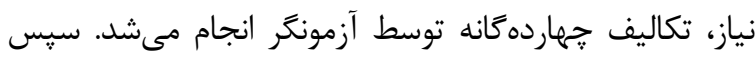

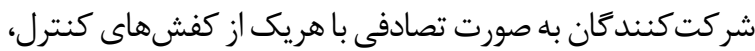

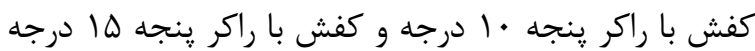

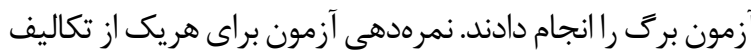

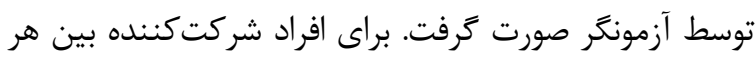
تعويض كفش ه دقيقه استر احت در نظر كرفته شد.

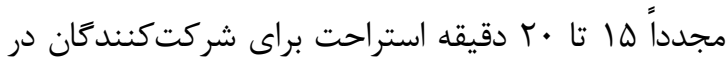

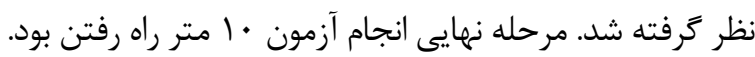

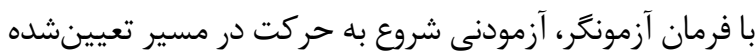

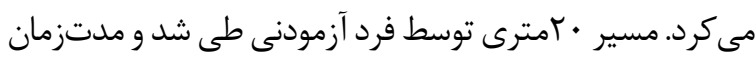

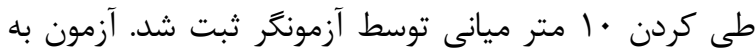

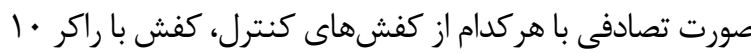

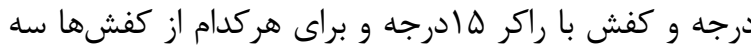

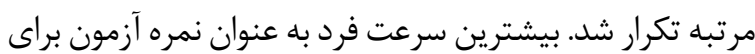
مداخله مشخص، ثبت شد.

به منظور تجزيهوتحليل دادهها، ابتدا توزيع كليه دادهها با

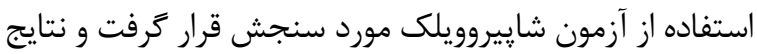

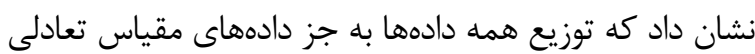

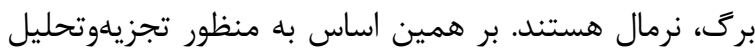

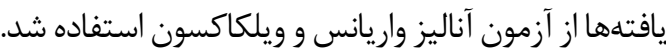

ياقتهها

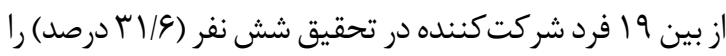
مردان و سا نفر (

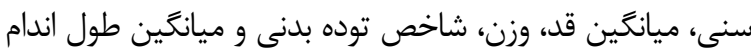

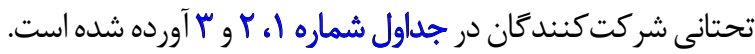

ميزان شاخص مقياس تعادلى برك در كروه كنترل

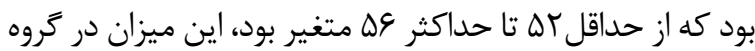

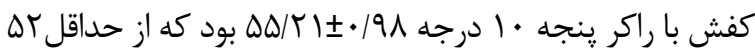

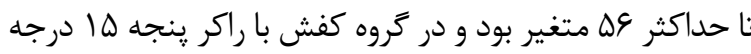

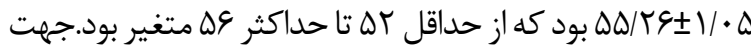

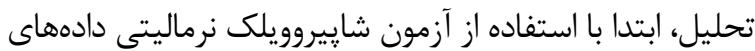

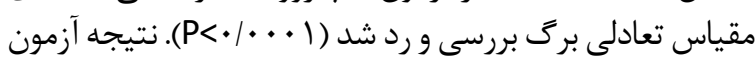


جدول ا. ميائكين توزيع كروه سنى

\begin{tabular}{|c|c|c|c|}
\hline درصد تجمعى & فراوانى (درصد) & يراكندمى & تروه سنى \\
\hline VNa & IO (NNQ) & . ع تا وه سال & \\
\hline $9 F / V$ & $r(1 Q / A)$ & r. r. & \\
\hline $1 .$. & $1(\Delta / r)$ & . A سال به بالا & \\
\hline- & $19(1 . .0)$ & مجموع & \\
\hline
\end{tabular}

سالمند سالم يرداخت نيز تفاوت قابل ملاحظهاى بين استفاده از

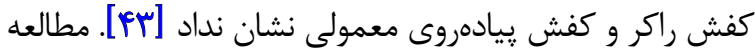

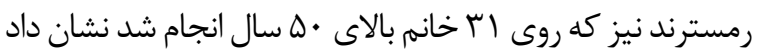

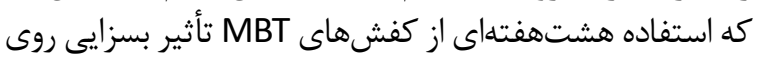

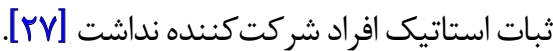
اين در حالى است كه نتايج مطالعات آلبرايت [FF]، دمورا

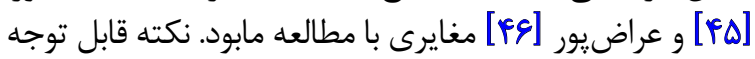
تفاوت نوع راكر و كفش مورداستفاده در هر سه مطالعه است. بيان

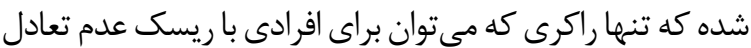

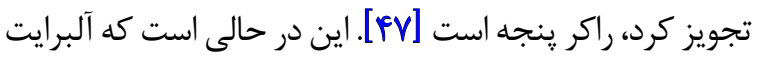

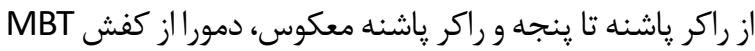

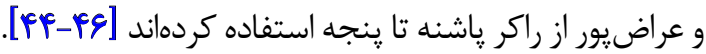
راكر پاشنه تا پنجه استفادهده در مطالعه آلبرايت به صورت

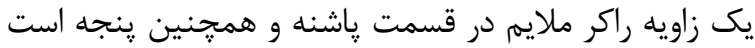

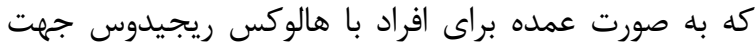

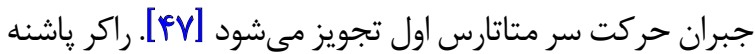

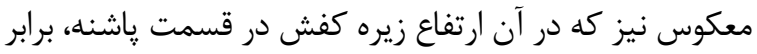

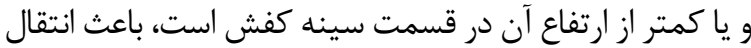

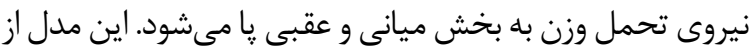

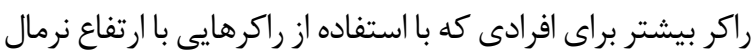

$$
\text { احساس بىثباتى مي كنند كاربرد دارد. }
$$

كفشهاى MBT استفادهشده در مطالعه دمورا داراى راكر

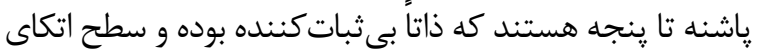

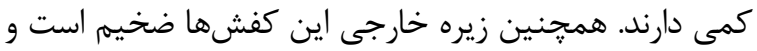
از جنسى منعطف در بخش ياشنه برخوردار هستند كه به به ديه دليل

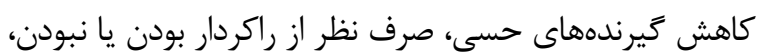

از اين تست در بررسى ثبات ياسجرال دايناميك رايج و يركاربرد

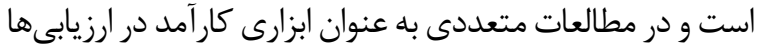

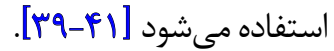

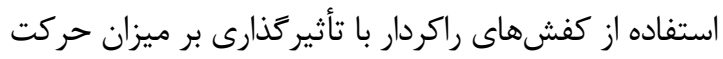

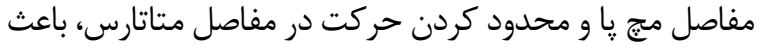

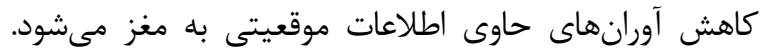

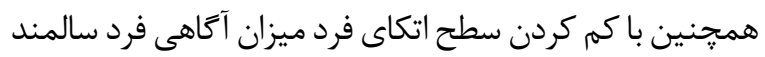

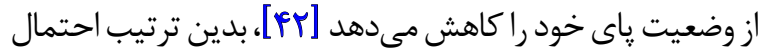

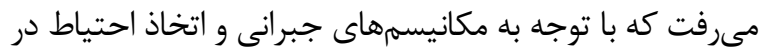

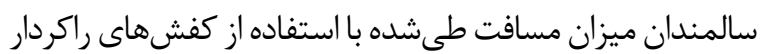

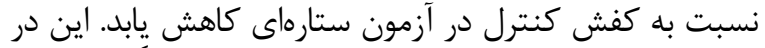

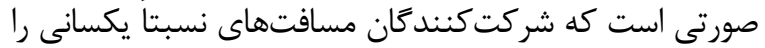

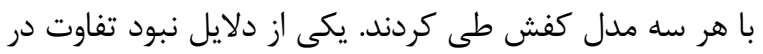

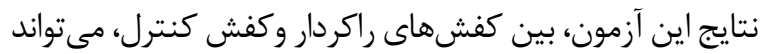

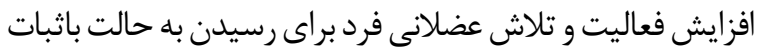

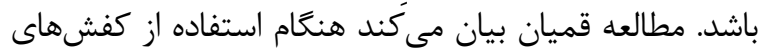

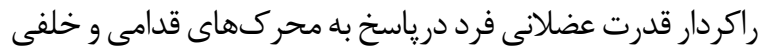

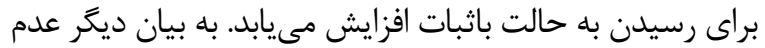

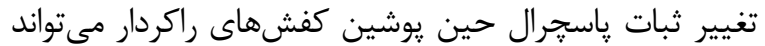

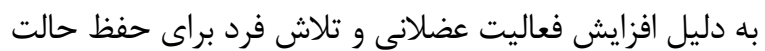
باثبات خود باشد [rها

مطالعه قميان روى هفده بيمار با نوروياتى ديابتى انجام شده

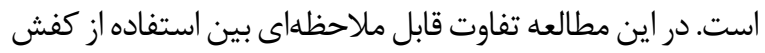

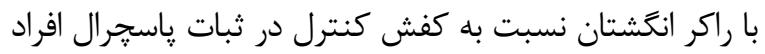

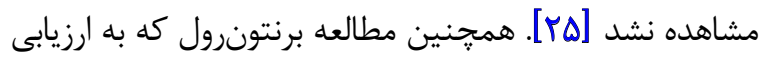

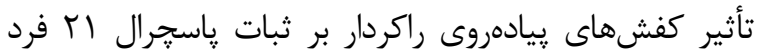

جدول ب. ميانكين قد، وزن و BMI

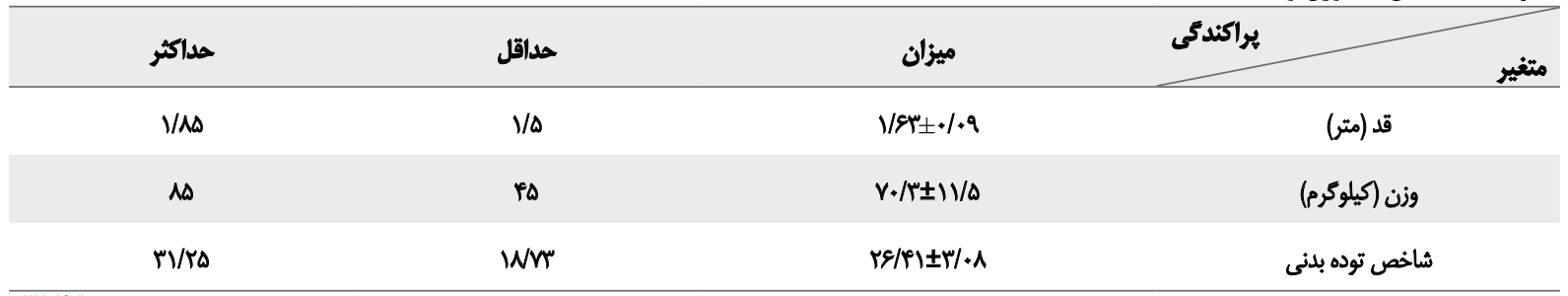


جدول"ّ. ميانكين طول ائدام تحتانى آزمودنىها

درصد تجمعي

$\Delta T / Y$

YNA

$A 9 / D$

$1 .$.

$-$
فراوانى (درصد)

$1+(\Delta T M)$

$1+(r \& / \pi)$

$r(1.10)$

$r(1 \cdot / \theta)$

$19(1.0)$
هيراكثدئى

طول اتذام تحتانيى

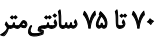

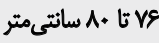

1 ا تا هم سانتىمثتر

ع ثا •9 سائتى متر

مجمن
نشان داد افزايش يا كاهش سرعت راه رفتن، با يوشيدن كفشهاى

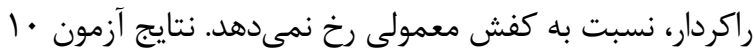

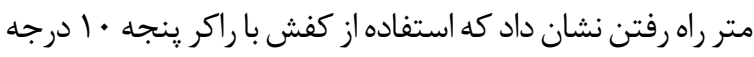

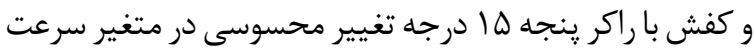
راه رفتن فرد ايجاد نمى كند.

افزودن راكر در زيره كفش باعث افزايش فعاليت عضلات

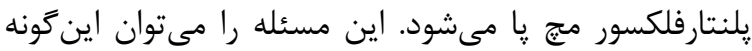

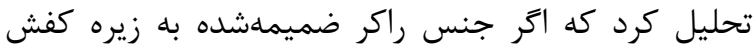

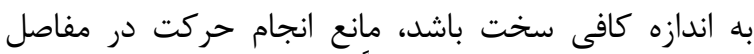

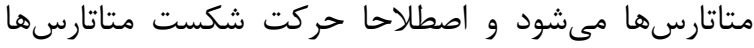

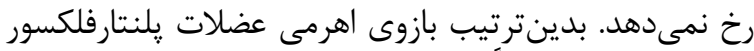

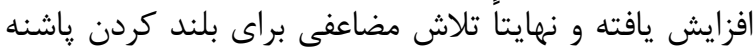

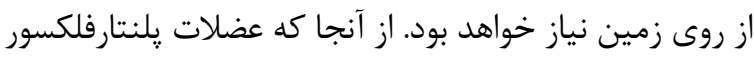

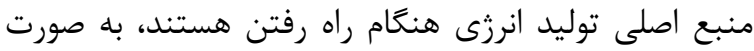

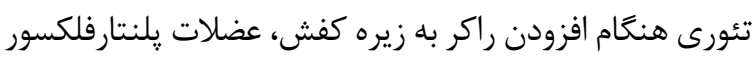

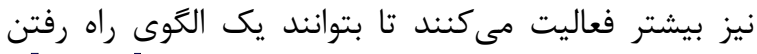

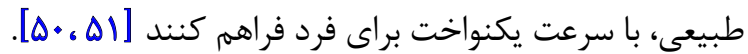

باعث به هم خوردن تعادل افراد مىشوند [FA. Fq]. مدل زيره

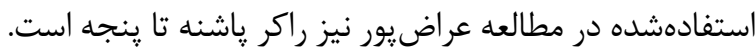
از طرفى ديخر رويه كفش استفادهده در در مطالعه عراض عراضيور

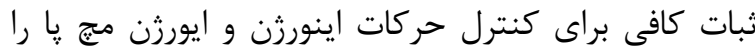

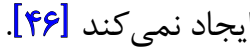
از طرفى ديخر تفاوت مشاهدهده بين يزوهش ما و مطالعات

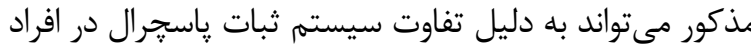

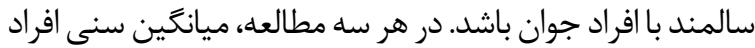

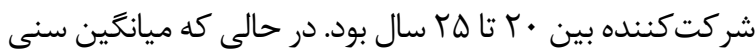
افراد شركت كننده مطالعه حاضر كو 9 سال است.

درنهايت از نقطه نظر ارزيابى تعادل در سالمندان سالم، يكى

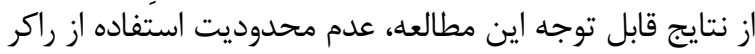

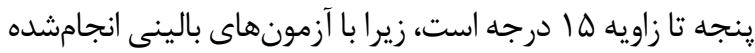

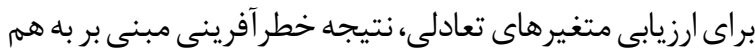

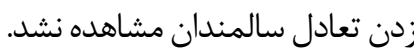

در رابطه با تأثير افزودن راكر ينجه به زيره كفش، مطالعه ما

جدول ع. شاخصهاى آزمون ستارهاى در سه راستاى مشخص

\begin{tabular}{|c|c|c|c|c|c|}
\hline تتيجه أزمون & راكر 10 درجه & راكر · ادرجه & كتترل & كروه & شاخص \\
\hline \multirow{3}{*}{$\mathrm{P}=$ / / } & $\cdot / \wedge \pm * / \cdot \gamma$ & $\cdot / M \pm .1 .9$ & $\cdot / Y A \pm \cdot / r$ & ميائكين +اتحرافسعيار & \multirow{3}{*}{ شائص آزمون ستارهاى در } \\
\hline & .189 & .180 & .18 & حكاقل & \\
\hline & 1 &.$/ 24$ & $V+F$ & حباكثر & \\
\hline \multirow{3}{*}{$\mathrm{P}=$ =/גזם } & $\cdot\left|\lambda r_{ \pm} \cdot /\right|$ & $\cdot / A r \pm \cdot / 11$ & $\cdot / \Lambda r_{ \pm} \cdot / \bullet \Lambda$ & مياتكين |نحرافمعيار & \multirow{3}{*}{ 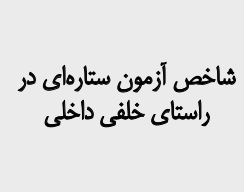 } \\
\hline & $\cdot M$ & . IAT & .199 & حداقل & \\
\hline & $1 / * r$ & $1 / \cdot 9$ & +/9Y & حلاكثر & \\
\hline \multirow{3}{*}{$P=* / \Delta 4$} & $\cdot \mid 8 q \pm \cdot / 11$ & $.180 \pm+/ 1$ & $. / F F \pm \cdot / 1 F$ & مياثكين +لاتحر افسمعيار & \multirow{3}{*}{ 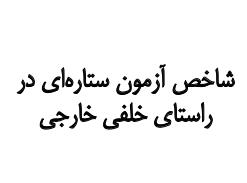 } \\
\hline &.$/ P 9$ & opr & $\cdot|r|$ & حباقل & \\
\hline &.$/ M$ &.$/ 94$ &.$/ 9 Y$ & حلاكثر & \\
\hline
\end{tabular}


ضخامت زيره بر تعادل مورد بحث است. از طرفى از آنجا كه

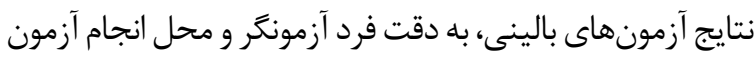

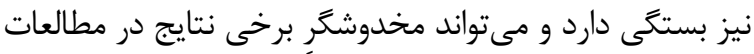

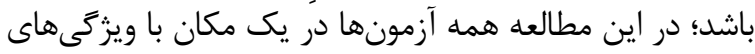

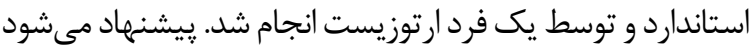

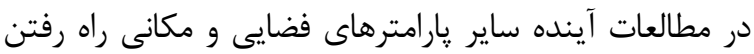

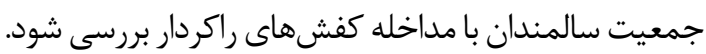

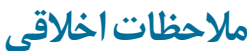

$$
\text { يبيروى از اصول اخلاق يزوهش }
$$

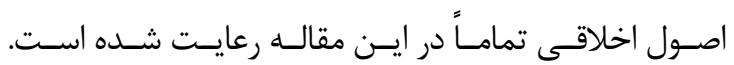

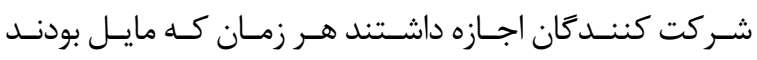

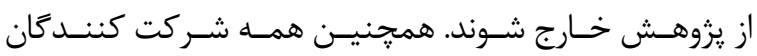

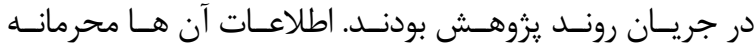

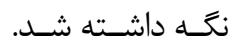

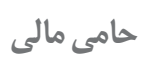

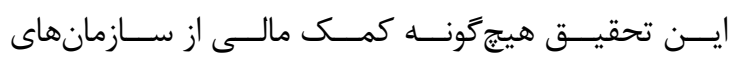

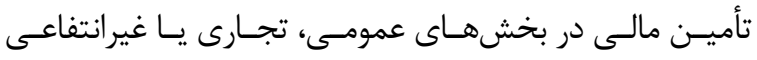

$$
\text { دريافـت نكـــــ }
$$

$$
\text { مشار كت نويسندكًان }
$$

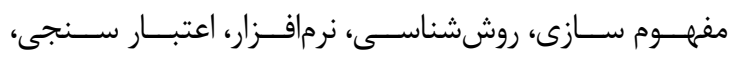

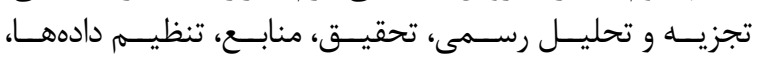

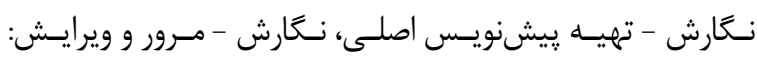

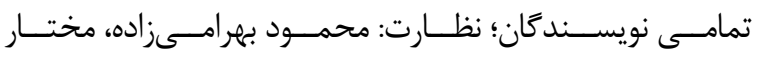

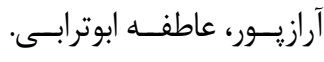

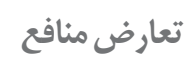

بنابر اظهار نويسندكان اين مقاله تعارض منافع ندارد.
از طرفى افزودن راكر ينجه به زيره كفش، باعث افزايش زاويه

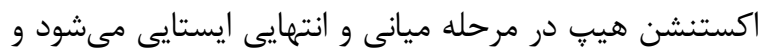

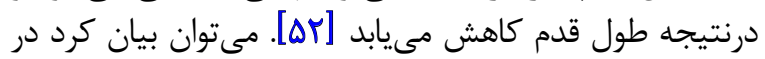

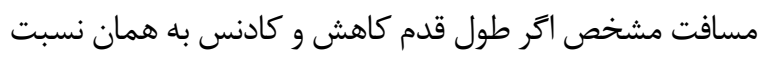
افزايش يابد، سرعت راه رفتن تغييرى نمى كند.

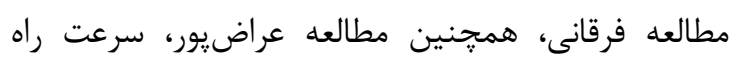

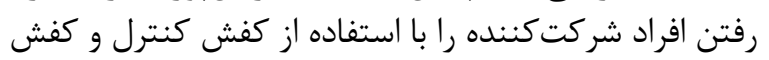

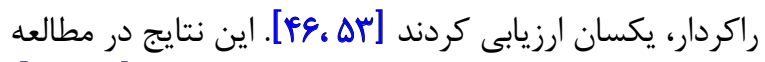

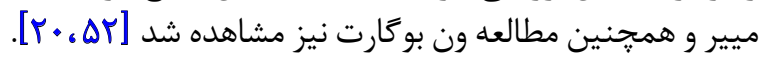

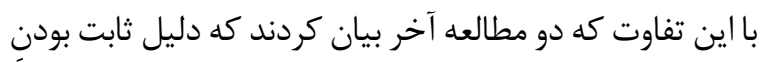

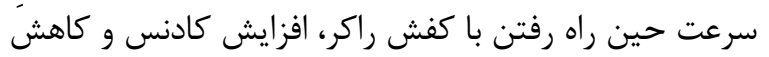

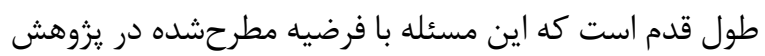

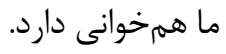

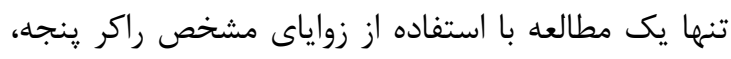

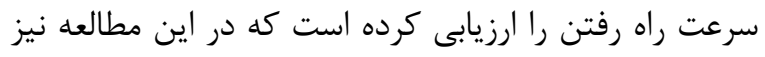

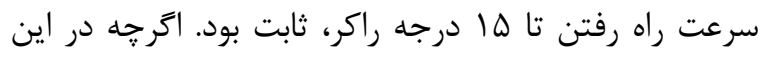

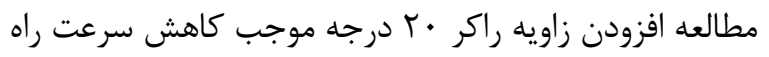

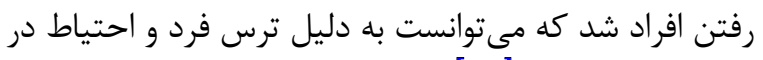

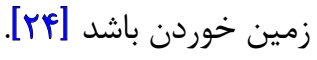

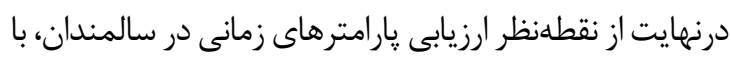

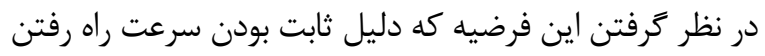

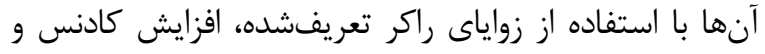

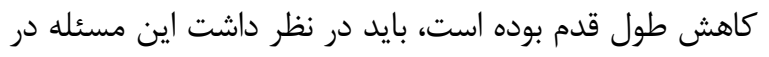

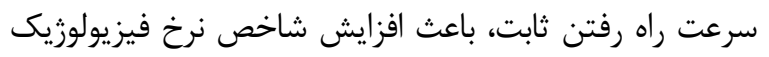

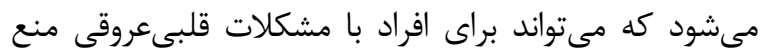

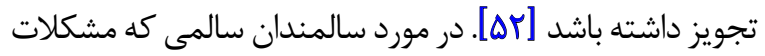

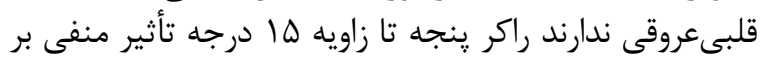

$$
\text { سرعت راه رفتن آنها ندارد. }
$$

\section{تثيجهُمَيرى}

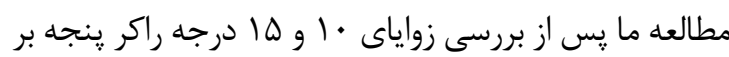

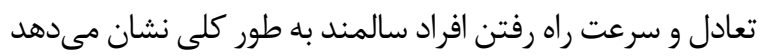

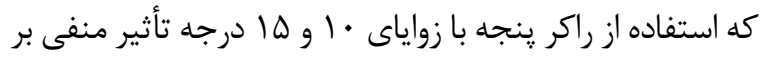

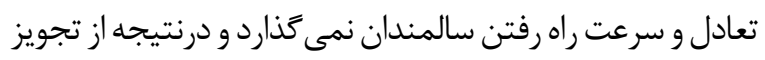

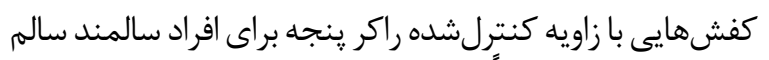

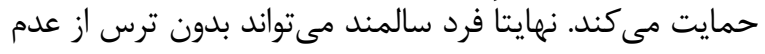

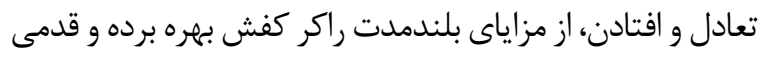
به سمت سالمندي يويا بردارد.

از نقطه نظر متغيرهاى مخدوشكر و محدوديتهاى اين مطالعه،

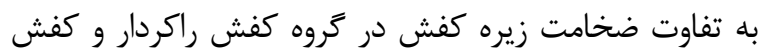

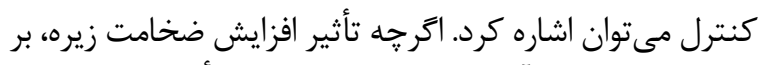
وزن كفشها از نظر آمارى نرمال شد، اما بازهم تأثير كذارى تفائ تفاوت 


\section{Referencs}

[1] Manton KG. Epidemiological, demographic, and social correlates of disability among the elderly. The Milbank Quarterly. 1989; 67(Suppl 2 Pt 1):13-58. [DOI:10.2307/3350235]

[2] Crimmins EM, Saito Y, Reynolds SL. Further evidence on recent trends in the prevalence and incidence of disability among older Americans from two sources: The LSOA and the NHIS. The Journals of Gerontology: Series B. 1997; 52B(2):S59-71. [DOI:10.1093/geronb/52B.2.S59]

[3] Diczfalusy E. The demographic revolution and our common future. Maturitas. 2001; 38(1):5-14. [DOI:10.1016/S03785122(00)00187-0]

[4] Benjuya N, Melzer I, Kaplanski J. Aging-induced shifts from a reliance on sensory input to muscle cocontraction during balanced standing. The Journals of Gerontology: Series A. 2004; 59(2):M166-71. [DOI:10.1093/gerona/59.2.M166]

[5] Mann R, Birks Y, Hall J, Torgerson D, Watt I. Exploring the relationship between fear of falling and neuroticism: Across-sectional study in community-dwelling women over 70 . Age and Ageing. 2006; 35(2):143-7. [DOI:10.1093/ageing/afj013]

[6] Hatch J, Gill-Body KM, Portney LG. Determinants of balance confidence in community-dwelling elderly people. Physical Therapy. 2003; 83(12):1072-9. [DOI:10.1093/ptj/83.12.1072]

[7] Arfken CL, Lach HW, Birge SJ, Miller JP. The prevalence and correlates of fear of falling in elderly persons living in the community. American Journal of Public Health. 1994; 84(4):565-70. [DOI:10.2105/AJPH.84.4.565]

[8] Sharaf AY, Ibrahim HS. Physical and psychosocial correlates of fear of falling among older adults in assisted living facilities. Journal of Gerontological Nursing. 2008; 34(12):27-35. [DOI:10.3928/00989134-20081201-07]

[9] Kulmala J, Sihvonen S, Kallinen M, Alen M, Kiviranta I, Sipilä $\mathrm{S}$. Balance confidence and functional balance in relation to falls in older persons with hip fracture history. Journal of Geriatric Physical Therapy. 2007; 30(3):114-20. [DOI:10.1519/00139143200712000-00006]

[10] Shumway-Cook A, Woollacott MH. Motor control: Translating research into clinical practice. Philadelphia: Lippincott Williams \& Wilkins; 2007. https://books.google.com/ books?id=BJcL3enz3xMC\&dq

[11] Lord SR, Ward JA, Williams Ph. Exercise effect on dynamic stability in older women: A randomized controlled trial. Archives of Physical Medicine and Rehabilitation. 1996; 77:232-6. [DOI:10.1016/S0003-9993(96)90103-3]

[12] Wallmann HW, Gillis CB, Alpert PT, Miller SK. The effect of a senior jazz dance class on static balance in healthy women over 50 years of age: A pilot study. Biological Research for Nursing. 2009; 10(3):257-66. [DOI:10.1177/1099800408322600]

[13] Brauer SG, Neros Ch, Woollacott M. Balance control in the elderly: Do Masters athletes show more efficient balance responses than healthy older adults? Aging Clinical and Experimental Research. 2008; 20(5):406-11. [DOI:10.1007/BF03325145]
[14] Xu DQ, Li JX, Hong Y. Effect of regular Tai Chi and jogging exercise on neuromuscular reaction in older people. Age and Ageing. 2005; 34(5):439-44. [DOI:10.1093/ageing/afi114]

[15] Studenski S, Duncan PW, Chandler J. Postural responses and effector factors in persons with unexplained falls: Results and methodologic issues. Journal of the American Geriatrics Society. 1991; 39(3):229-34. [DOI:10.1111/j.1532-5415.1991.tb01642.x]

[16] Hijmans JM, Geertzen JHB, Dijkstra PU, Postema K. A systematic review of the effects of shoes and other ankle or foot appliances on balance in older people and people with peripheral nervous system disorders. Gait \& Posture. 2007; 25(2):316-23. [DOI:10.1016/j.gaitpost.2006.03.010]

[17] Perry SD, Radtke A, Goodwin CR. Influence of footwear midsole material hardness on dynamic balance control during unexpected gait termination. Gait \& Posture. 2007; 25(1):94-8. [DOI:10.1016/i. gaitpost.2006.01.005

[18] Wilson ML, Rome K, Hodgson D, Ball P. Effect of textured foot orthotics on static and dynamic postural stability in middle-aged females. Gait \& Posture. 2008; 27(1):36-42. [DOI:10.1016/j.gaitpost.2006.12.006]

[19] Brown D, Wertsch JJ, Harris GF, Klein J, Janisse D. Effect of rocker soles on plantar pressures. Archives of Physical Medicine and Rehabilitation. 2004; 85(1):81-6. [DOI:10.1016/S00039993(03)00374-5]

[20] Meyer PF, Oddsson LIE, De Luca CJ. The role of plantar cutaneous sensation in unperturbed stance. Experimental Brain Research 2004; 156(4):505-12. [DOI:10.1007/s00221-003-1804-y]

[21] van Schie C, Ulbrecht JS, Becker MB, Cavanagh PR. Design criteria for rigid rocker shoes. Foot \& Ankle International. 2000; 21(10):83344. [DOI:10.1177/107110070002101007]

[22] Myers KA, Long JT, Klein JP, Wertsch JJ, Janisse D, Harris GF Biomechanical implications of the negative heel rocker sole shoe: Gait kinematics and kinetics. Gait \& Posture. 2006; 24(3):323-30. DOI:10.1016/i.gaitpost.2005.10.006]

[23] Coughlan GF, Fullam K, Delahunt E, Gissane C, Caulfield BM. A comparison between performance on selected directions of the star excursion balance test and the $\mathrm{Y}$ balance test. Journal of Athletic Training. 2012; 47(4):366-71. [DOI:10.4085/1062-6050-47.4.03]

[24] Thies SB, Jones RK, Kenney LPJ, Howard D, Baker R. Effects of ramp negotiation, paving type and shoe sole geometry on toe clearance in young adults. Journal of Biomechanics. 2011; 44(15):267984. [DOI:10.1016/j.jbiomech.2011.07.027]

[25] Ghomian B, Kamyab M, Jafari H, Khamseh ME, Healy A. Rocker outsole shoe is not a threat to postural stability in patients with diabetic neuropathy. Prosthetics and Orthotics International. 2016; 40(2):224-30. [DOI:10.1177/0309364614543549]

[26] Sousa ASP, Silva A, Macedo R, Santos R, Tavares JMRS. Influence of long-term wearing of unstable shoes on compensatory control of posture: An electromyography-based analysis. Gait \& Posture. 2014; 39(1):98-104. [DOI:10.1016/j.gaitpost.2013.06.003]

[27] Ramstrand N, Thuesen AH, Nielsen DB, Rusaw D. Effects of an unstable shoe construction on balance in women aged over 50 years. Clinical Biomechanics. 2010; 25(5):455-60. [DOI:10.1016/j.clinbiomech.2010.01.014] 
[28] Landry SC, Nigg BM, Tecante KE. Standing in an unstable shoe increases postural sway and muscle activity of selected smaller extrinsic foot muscles. Gait \& Posture. 2010; 32(2):215-9. [DOI:10.1016/j.gaitpost.2010.04.018]

[29] Gribble PA, Hertel J. Considerations for normalizing measures of the Star Excursion Balance Test. Measurement in Physical Education and Exercise Science. 2003; 7(2):89-100. [DOI:10.1207/ S15327841MPEE0702_3]

[30] Hertel J, Miller SJ, Denegar CR. Intratester and intertester reliability during the Star Excursion Balance Tests. Journal of Sport Rehabilitation. 2000; 9(2):104-16. [DOI:10.1123/jsr.9.2.104]

[31] Salavati M, Negahban H, Mazaheri M, Soleimanifar M, Hadadi M, Sefiddashti L, et al. The Persian version of the Berg Balance Scale: Inter and intra-rater reliability and construct validity in elderly adults. Disability and Rehabilitation. 2012;34(20):1695-8. [D OI:10.3109/09638288.2012.660604]

[32] Bogle Thorbahn LD, Newton RA. Use of the Berg Balance Test to predict falls in elderly persons. Physical Therapy. 1996; 76(6):576-83. [DOI:10.1093/ptj/76.6.576]

[33] Peters DM, Fritz SL, Krotish DE. Assessing the reliability and validity of a shorter walk test compared with the 10-Meter Walk Test for measurements of gait speed in healthy, older adults. Journal of Geriatric Physical Therapy. 2013; 36(1):24-30. [DOI:10.1519/JPT.0b013e318248e20d]

[34] Robbins S, Waked E, Gouw GJ, McClaran J. Athletic footwear affects balance in men. British Journal of Sports Medicine. 1994; 28(2):117-22. [DOI:10.1136/bjsm.28.2.117]

[35] Thies SB, Price C, Kenney LPJ, Baker R. Effects of shoe sole geometry on toe clearance and walking stability in older adults. Gait \& Posture. 2015; 42(2):105-9. [DOI:10.1016/j.gaitpost.2015.04.011]

[36] Hadadi M, Mazaheri M, Mousavi ME, Maroufi N, Bahramizadeh M, Fardipour Sh. Effects of soft and semi-rigid ankle orthoses on postural sway in people with and without functional ankle instability. Journal of Science and Medicine in Sport. 2011; 14(5):370-5. [DOI:10.1016/j.jsams.2010.12.004]

[37] Wikstrom EA, Arrigenna MA, Tillman MD, Borsa PA. Dynamic postural stability in subjects with braced, functionally unstable ankles. Journal of Athletic Training. 2006; 41(3):245-50. [PMID] [PMCID]

[38] Kinzey SJ, Armstrong CW. The reliability of the star-excursion test in assessing dynamic balance. Journal of Orthopaedic \& Sports Physical Therapy. 1998; 27(5):356-60. [DOI:10.2519/ jospt.1998.27.5.356]

[39] Hardy L, Huxel K, Brucker J, Nesser T. Prophylactic ankle braces and star excursion balance measures in healthy volunteers. Journal of Athletic Training. 2008; 43(4):347-51. [DOI:10.4085/10626050-43.4.347]

[40] Hadadi M, Mousavi ME, Fardipour Sh, Vameghi R, Mazaheri M. Effect of soft and semirigid ankle orthoses on Star Excursion Balance Test performance in patients with functional ankle instability. Journal of Science and Medicine in Sport. 2014; 17(4):4303. [DOI:10.1016/j.jsams.2013.05.017]
[41] Sesma AR, Mattacola CG, Uhl TL, Nitz AJ, McKeon PO. Effect of foot orthotics on single-and double-limb dynamic balance tasks in patients with chronic ankle instability. Foot \& Ankle Specialist. 2008; 1(6):330-7. [DOI:10.1177/1938640008327516]

[42] Robbins S, Waked E, Allard P, McClaran J, Krouglicof N. Foot position awareness in younger and older men: The influence of footwear sole properties. Journal of the American Geriatrics Society. 1997; 45(1):61-6. [DOI:10.1111/j.1532-5415.1997.tb00979.x]

[43] Brenton-Rule A, Bassett S, Walsh A, Rome K. The evaluation of walking footwear on postural stability in healthy older adults: An exploratory study. Clinical Biomechanics. 2011; 26(8):885-7. [DOI:10.1016/j.clinbiomech.2011.03.012]

[44] Albright BC, Woodhull-Smith WM. Rocker bottom soles alter the postural response to backward translation during stance. Gait \& Posture. 2009; 30(1):45-9. [DOI:10.1016/j.gaitpost.2009.02.012]

[45] Demura T, Demura SI, Uchiyama M, Kitabayashi T, Takahashi $\mathrm{K}$. Effect of shoes with rounded soft soles in the anterior-posterior direction on the center of pressure during static standing. The Foot. 2015; 25(2):97-100. [DOI:10.1016/j.foot.2015.02.004]

[46] Arazpour M, Hutchins SW, Ghomshe FT, Shaky F, Karami MV, Aksenov AY. Effects of the heel-to-toe rocker sole on walking in able-bodied persons. Prosthetics and Orthotics International. 2013; 37(6):429-35. [DOI:10.1177/0309364612474920]

[47] Janisse DJ. Shoes and shoe modifications. In: Hsu JD, Michael JW, Fisk JR, editors. AAOS Atlas of Orthoses and Assistive Devices. $4^{\text {th }}$ ed. Philadelphia, PA: Mosby; 2008. pp. 325-334. https:// books.google.com/books?id $=$ Q738LYIlcR8C $\&$ printsec $=$ frontc over\&dq

[48] Waked E, Robbins S, McClaran J. The effect of footwear midsole hardness and thickness on proprioception and stability in older men. Journal of Testing and Evaluation. 1997; 25(1):143-8. [DOI:10.1520/JTE11335J]

[49] Robbins S, Gouw GJ, McClaran J. Shoe sole thickness and hardness influence balance in older men. Journal of the American Geriatrics Society. 1992; 40(11):1089-94. [DOI:10.1111/j.1532-5415.1992.tb01795.x]

[50] Grundy M, Tosh PA, McLeish RD, Smidt L. An investigation of the centres of pressure under the foot while walking. The Journal of Bone and Joint Surgery. 1975; 57-B(1):98-103. [DOI:10.1302/0301-620X.57B1.98]

[51] Harris G, Klein J, Janisse D, Brown D, Shu Y, Wertsch J. Effect of rocker-soles on lower extremity dynamic EMG patterns. Gait Posture. 2000; 11:157-8.

[52] Van Bogart JJ, Long JT, Klein JP, Wertsch JJ, Janisse DJ, Harris GF. Effects of the toe-only rocker on gait kinematics and kinetics in able-bodied persons. IEEE Transactions on Neural Systems and Rehabilitation Engineering. 2005; 13(4):542-50. [DOI:10.1109/TNSRE.2005.858460]

[53] Forghany S, Nester CJ, Richards B, Hatton AL, Liu A. Rollover footwear affects lower limb biomechanics during walking. Gait \& Posture. 2014; 39(1):205-12. [DOI:10.1016/j.gaitpost.2013.07.009] 